\title{
Improved Bounds on the Average Distance to the Fermat-Weber Center of a Convex Object
}

\author{
A. Karim Abu-Affash*
}

\author{
Matthew J. Katz*
}

\begin{abstract}
We show that for any convex object $Q$ in the plane, the average distance between the Fermat-Weber center of $Q$ and the points in $Q$ is at least $4 \Delta(Q) / 25$, and at most $2 \Delta(Q) /(3 \sqrt{3})$, where $\Delta(Q)$ is the diameter of $Q$. We use the former bound to improve the approximation ratio of a load-balancing algorithm of Aronov et al. [1].
\end{abstract}

\section{Introduction}

The Fermat-Weber center of an object $Q$ in the plane is a point in the plane, such that the average distance from it to the points in $Q$ is minimal. For an object $Q$ and a point $y$, let $\mu_{Q}(y)$ be the average distance between $y$ and the points in $Q$, that is, $\mu_{Q}(y)=\int_{x \in Q}\|x y\| d x / \operatorname{area}(Q)$, where $\|x y\|$ is the Euclidean distance between $x$ and $y$. Let $\mathcal{F} \mathcal{W}_{Q}$ be a point for which this average distance is minimal, that is, $\mu_{Q}\left(\mathcal{F} \mathcal{W}_{Q}\right)=\min _{y} \mu_{Q}(y)$, and put $\mu_{Q}^{*}=\mu_{Q}\left(\mathcal{F} \mathcal{W}_{Q}\right)$. The point $\mathcal{F} \mathcal{W}_{Q}$ is a Fermat-Weber center of $Q$.

It is easy to verify, for example, that the FermatWeber center of a disk $D$ coincides with the center $o$ of $D$, and that the average distance between $o$ and the points in $D$ is $\Delta(D) / 3$, where $\Delta(D)$ is the diameter of $D$. Carmi, Har-Peled, and Katz [3] studied the relation between $\mu_{Q}^{*}$ and the diameter of $Q$, denoted $\Delta(Q)$. They proved that there exists a constant $c_{1}$, such that, for any convex object $Q$, the average distance between a Fermat-Weber center of $Q$ and the points in $Q$ is at least $c_{1} \Delta(Q)$, and that the largest such constant $c_{1}^{*}$ lies in the range $[1 / 7 . .1 / 6]$.

In this paper, we both improve the above bound on $c_{1}^{*}$, and tightly bound a new constant $c_{2}^{*}$; see below. More precisely, we first significantly narrow the range in which $c_{1}^{*}$ must lie, by proving (in Section 2) that $4 / 25 \leq c_{1}^{*} \leq 1 / 6$. Next, we consider the question what is the smallest constant $c_{2}^{*}$, such that, for any convex object $Q, \mu_{Q}^{*} \leq c_{2}^{*} \Delta(Q)$. We prove (in Section 3 ) that $1 / 3 \leq c_{2}^{*} \leq 2 /(3 \sqrt{3})$. A useful corollary obtained from these results is that the average distance to the center

*Department of Computer Science, Ben-Gurion University, Israel; \{abuaffas, matya\}@cs.bgu.ac.il. A.K. Abu-Affash was partially supported by the Lynn and William Frankel Center for Computer Sciences. of the smallest enclosing circle of a convex $n$-gon $P$ is less than 2.41 times $\mu_{P}^{*}$.

The Fermat-Weber center of an object $Q$ is a very significant point. The classical Fermat-Weber problem is: Find a point in a set $F$ of feasible facility locations, that minimizes the average distance to the points in a set $D$ of (possibly weighted) demand locations. If $D$ is a finite set of points, $F$ is the entire plane, and distances are measured using the $L_{2}$ metric, then it is known that the solution is algebraic [2]. See Wesolowsky [8] for a survey of the Fermat-Weber problem.

Only a few papers deal with the continuous version of the Fermat-Weber problem, where the set of demand locations is continuous. Fekete, Mitchell and Weinbrecht [4] presented algorithms for computing an optimal solution for $D=F=P$ where $P$ is a simple polygon or a polygon with holes, and the distance between two points in $P$ is the $L_{1}$ geodesic distance between them. Carmi, Har-Peled and Katz [3] presented a linear-time approximation scheme for the case where $P$ is a convex polygon.

Aronov et al. [1] considered the following load balancing problem. Let $D$ be a convex region and let $\mathcal{P}=\left\{p_{1}, \ldots, p_{m}\right\}$ be a set of $m$ points representing $m$ facilities. One would like to divide $D$ into $m$ equal-area subregions $R_{1}, \ldots, R_{m}$, so that region $R_{i}$ is associated with point $p_{i}$, and the total cost of the subdivision is minimized. Given a subdivision, the cost $\kappa\left(p_{i}\right)$ associated with facility $p_{i}$ is the average distance between $p_{i}$ and the points in $R_{i}$, and the total cost of the subdivision is $\sum_{i} \kappa\left(p_{i}\right)$.

Aronov, et al. discussed the structure of an optimal subdivision, and also presented an $(8+\sqrt{2 \pi})$ approximation algorithm, under the assumption that the regions $R_{1}, \ldots, R_{m}$ must be convex and that $D$ is a rectangle. Our improved bound on the constant $c_{1}^{*}$, allows us (in Section 4) to improve the above approximation ratio.

\section{$24 / 25 \leq c_{1}^{*} \leq 1 / 6$}

Carmi, Har-Peled and Katz [3] showed that there exists a convex polygon $P$ such that $\mu_{P}^{*} \leq \Delta(P) / 6$. This immediately implies that $c_{1}^{*} \leq 1 / 6$. We prove below that $c_{1}^{*} \geq 4 / 25$. Our proof is similar in its structure to the proof of $[3]$. 
Theorem 2.1. Let $P$ be a convex object. Then $\mu_{P}^{*} \geq$ $4 \Delta(P) / 25$.

Proof: Let $\mathcal{F W}_{P}$ be a Fermat-Weber center of $P$. We need to show that $\int_{x \in P}\left\|x \mathcal{F} \mathcal{W}_{P}\right\| d x \geq \frac{4 \Delta(P)}{25}$ area $(P)$. We do this in two stages. In the first stage we show that for a certain subset $P^{\prime}$ of $P, \int_{x \in P^{\prime}}\left\|x \mathcal{F} \mathcal{W}_{P}\right\| d x \geq$ $\frac{4 \Delta(P)}{27}$ area $(P)$. This implies that for any convex object $Q, \mu_{Q}^{*} \geq 4 \Delta(Q) / 27$. In the second stage we apply this intermediate result to a collection of convex subsets of $P-P^{\prime}$ that are pairwise disjoint to obtain the claimed result. This latter stage is essentially identical to the second stage in the proof of [3]; it is included here for the reader's convenience.

We now describe the first stage. Let $s$ be a line segment of length $\Delta(P)$ connecting two points $p$ and $q$ on the boundary of $P$. We may assume that $s$ is horizontal and that $p$ is its left endpoint, since one can always rotate $P$ around, say, $p$ until this is the case.

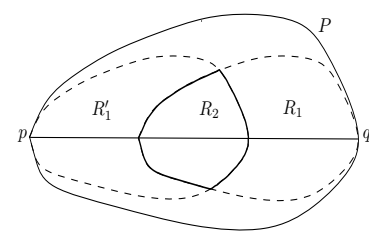

(a)

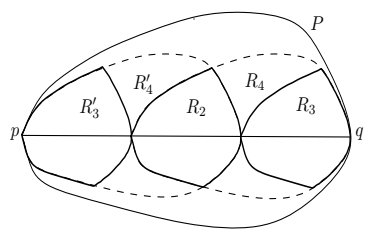

(b)
Figure 1: Proof of intermediate result.

Let $P^{\alpha}$ be the polygon obtained from $P$ by shrinking it by a factor of $\alpha$, that is, by applying the transformation $f(a, b)=(a / \alpha, b / \alpha)$ to the points $(a, b)$ in $P$. We place a copy $R_{1}$ of $P^{3 / 2}$, such that $R_{1}$ is contained in $P$ and has a common tangent with $P$ at $q$. Similarly, we place a copy $R_{1}^{\prime}$ of $P^{3 / 2}$, such that $R_{1}^{\prime}$ is contained in $P$ and has a common tangent with $P$ at $p$; see Figure 1(a). Clearly, area $\left(R_{1}\right)=\operatorname{area}\left(R_{1}^{\prime}\right)=\frac{4}{9} \operatorname{area}(P)$.

Let $R_{2}=R_{1} \cap R_{1}^{\prime}$. We place a copy $R_{3}$ of $R_{2}$, such that $R_{3}$ is contained in $R_{1}$ and has a common tangent with $R_{1}$ at $q$. Similarly, we place a copy $R_{3}^{\prime}$ of $R_{2}$, such that $R_{3}^{\prime}$ is contained in $R_{1}^{\prime}$ and has a common tangent with $R_{1}^{\prime}$ at $p$. Let $R_{4}=R_{1}-\left(R_{2} \cup R_{3}\right)$ and $R_{4}^{\prime}=R_{1}^{\prime}-\left(R_{2} \cup R_{3}^{\prime}\right)$; see Figure $1(\mathrm{~b})$.

We know that, regardless of the exact location of $\mathcal{F} \mathcal{W}_{P}$, the distance between $\mathcal{F} \mathcal{W}_{P}$ and the points in $R_{3}$ plus the distance between $\mathcal{F} \mathcal{W}_{P}$ and the points in $R_{3}^{\prime}$ is greater than $\frac{2 \Delta(P)}{3}$ area $\left(R_{3}\right)$, and the distance between $\mathcal{F W}_{P}$ and the points in $R_{4}$ plus the distance between $\mathcal{F W}_{P}$ and the points in $R_{4}^{\prime}$ is greater than $\frac{\Delta(P)}{3}$ area $\left(R_{4}\right)$. More precisely,

$\int_{x \in R_{3}}\left\|x \mathcal{F} \mathcal{W}_{P}\right\| d x+\int_{x \in R_{3}^{\prime}}\left\|x \mathcal{F} \mathcal{W}_{P}\right\| d x \geq \frac{2 \Delta(P)}{3} \operatorname{area}\left(R_{3}\right)$ and

$\int_{x \in R_{4}}\left\|x \mathcal{F} \mathcal{W}_{P}\right\| d x+\int_{x \in R_{4}^{\prime}}\left\|x \mathcal{F} \mathcal{W}_{P}\right\| d x \geq \frac{\Delta(P)}{3} \operatorname{area}\left(R_{4}\right)$.

Since area $\left(R_{4}\right)=\operatorname{area}\left(R_{1}\right)-\left(\operatorname{area}\left(R_{2}\right) \cup \operatorname{area}\left(R_{3}\right)\right)=$ $\frac{4}{9}$ area $(P)-2$ area $\left(R_{3}\right)$, we obtain our intermediate result

$$
\begin{aligned}
\int_{x \in P} & \left\|x \mathcal{F} \mathcal{W}_{P}\right\| d x \geq \int_{x \in R_{3}}\left\|x \mathcal{F} \mathcal{W}_{P}\right\| d x+ \\
& +\int_{x \in R_{3}^{\prime}}\left\|x \mathcal{F} \mathcal{W}_{P}\right\| d x+\int_{x \in R_{4}}\left\|x \mathcal{F} \mathcal{W}_{P}\right\| d x+ \\
& +\int_{x \in R_{4}^{\prime}}\left\|x \mathcal{F} \mathcal{W}_{P}\right\| d x \geq \frac{2 \Delta(P)}{3} \operatorname{area}\left(R_{3}\right)+ \\
& +\frac{\Delta(P)}{3}\left(\frac{4}{9} \operatorname{area}(P)-2 \operatorname{area}\left(R_{3}\right)\right)=\frac{4 \Delta(P)}{27} \operatorname{area}(P) .
\end{aligned}
$$

This intermediate result immediately implies that for any convex object $Q, \mu_{Q}^{*} \geq 4 \Delta(Q) / 27$. In the second stage we show that the 27 in the denominator can be replaced by 25 .

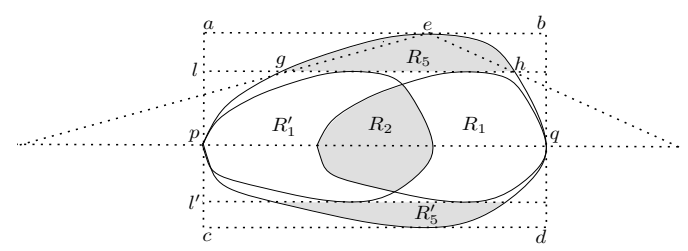

Figure 2: Proof of improved result.

Consider Figure 2. We draw the axis-aligned bounding box of $P$. The line segment $s$ (whose length is $\Delta(P)$ ) divides the bounding box of $P$ into two rectangles, $a b q p$ above $s$ and $p q d c$ below $s$. We divide each of these rectangles into two parts (a lower part and an upper part), by drawing the two horizontal lines $l$ and $l^{\prime}$. Let $R_{5}$ denote the intersection of $P$ with the upper part of the upper rectangle, and let $R_{5}^{\prime}$ denote the intersection of $P$ with the lower part of the lower rectangle.

Let $e$ be any point on the segment $a b$ that also lies on the boundary of $R_{5}$. We mention several facts concerning $R_{5}$ and $R_{5}^{\prime} . R_{5} \cap R_{5}^{\prime}=\phi, R_{5} \cap R_{1}=\phi, R_{5} \cap R_{1}^{\prime}=\phi$, $R_{5}^{\prime} \cap R_{1}=\phi$, and $R_{5}^{\prime} \cap R_{1}^{\prime}=\phi$. Notice also that $\Delta\left(R_{5}\right)$, $\Delta\left(R_{5}^{\prime}\right) \geq \Delta(P) / 3$, since, e.g., the line segment $l \cap R_{5}$ contains the base of the triangle that is obtained by intersecting the triangle $p e q$ with $R_{5}$, and the length of this base is $\Delta(P) / 3$.

We observe that $\operatorname{area}\left(R_{5}\right)+\operatorname{area}\left(R_{5}^{\prime}\right) \geq \operatorname{area}(P) / 9$ by showing that area $\left(R_{5}\right) \geq \operatorname{area}(P \cap a b q p) / 9$ (and that $\left.\operatorname{area}\left(R_{5}^{\prime}\right) \geq \operatorname{area}(P \cap p q d c) / 9\right)$. Let $g, h$ be the two points on the line $l$ that also lie on the boundary of $R_{5}$. Let $l(s)$ be the line containing $s$, and let $T$ be the triangle defined by $l(s)$ and the two line segments connecting $e$ to $l(s)$ and passing through $g$ and through $h$, respectively. Let $T_{2}$ denote the triangle $g e h$. 
Clearly $T_{2} \subseteq R_{5}$. Put $Q=R_{5}-T_{2}$. Then, $\operatorname{area}\left(R_{5}\right)=$ area $\left(T_{2}\right)+\operatorname{area}(Q)=\operatorname{area}(T) / 9+\operatorname{area}(Q)$. Therefore, $\operatorname{area}\left(R_{5}\right) \geq(\operatorname{area}(T)+\operatorname{area}(Q)) / 9 \geq \operatorname{area}(P \cap a b q p) / 9$. We show that area $\left(R_{5}^{\prime}\right) \geq \operatorname{area}(P \cap p q d c) / 9$ using the "symmetric" construction. Since $(P \cap a b q p) \cup(P \cap$ $p q d c)=P$ we obtain that $\operatorname{area}\left(R_{5}\right)+\operatorname{area}\left(R_{5}^{\prime}\right) \geq$ area $(P) / 9$.

It is also easy to see that $\Delta\left(R_{2}\right)=\Delta(P) / 3$ and area $\left(R_{2}\right) \geq \operatorname{area}(P) / 9$. This is because $P^{3} \subseteq R_{2}$ and area $\left(P^{3}\right)=\operatorname{area}(P) / 9$, where $P^{3}$ is the polygon obtained from $P$ by shrinking it by a factor of 3 .

Now using the implication of our intermediate result we have

$$
\begin{aligned}
\int_{x \in R_{5}}\left\|x \mathcal{F} \mathcal{W}_{P}\right\| d x+\int_{x \in R_{5}^{\prime}}\left\|x \mathcal{F} \mathcal{W}_{P}\right\| d x+ \\
\quad+\int_{x \in R_{2}}\left\|x \mathcal{F} \mathcal{W}_{P}\right\| d x \geq \frac{4 \Delta\left(R_{5}\right)}{27} \operatorname{area}\left(R_{5}\right)+ \\
\quad+\frac{4 \Delta\left(R_{5}^{\prime}\right)}{27} \operatorname{area}\left(R_{5}^{\prime}\right)+\frac{4 \Delta\left(R_{2}\right)}{27} \operatorname{area}\left(R_{2}\right) \geq \\
\quad \geq \frac{4 \Delta(P)}{81}\left(\operatorname{area}\left(R_{5}\right)+\operatorname{area}\left(R_{5}^{\prime}\right)+\operatorname{area}\left(R_{2}\right)\right) \geq \\
\geq \frac{8 \Delta(P)}{729} \operatorname{area}(P) .
\end{aligned}
$$

Therefore

$$
\begin{aligned}
\int_{x \in P} & \left\|x \mathcal{F} \mathcal{W}_{P}\right\| d x \geq \int_{x \in R_{3}}\left\|x \mathcal{F} \mathcal{W}_{P}\right\| d x+ \\
& +\int_{x \in R_{3}^{\prime}}\left\|x \mathcal{F} \mathcal{W}_{P}\right\| d x+\int_{x \in R_{4}}\left\|x \mathcal{F} \mathcal{W}_{P}\right\| d x+ \\
& +\int_{x \in R_{4}^{\prime}}\left\|x \mathcal{F} \mathcal{W}_{P}\right\| d x+\int_{x \in R_{5}}\left\|x \mathcal{F} \mathcal{W}_{P}\right\| d x+ \\
& +\int_{x \in R_{5}^{\prime}}\left\|x \mathcal{F} \mathcal{W}_{P}\right\| d x+\int_{x \in R_{2}}\left\|x \mathcal{F} \mathcal{W}_{P}\right\| d x \geq \\
\geq & \frac{4 \Delta(P)}{27} \operatorname{area}(P)+\frac{8 \Delta(P)}{729} \operatorname{area}(P)= \\
= & \frac{116 \Delta(P)}{729} \operatorname{area}(P) .
\end{aligned}
$$

At this point we may conclude that for any convex object $Q, \mu_{Q}^{*} \geq 116 \Delta(Q) / 729$. So we repeat the calculation above using this result for the regions $R_{5}, R_{5}^{\prime}$ and $R_{2}$ (instead of using the slightly weaker result, i.e., $\left.\mu_{Q}^{*} \geq 4 \Delta(Q) / 27\right)$. This calculation will yield a slightly stronger result, etc. In general, the result after the $k$-th iteration is $\mu_{Q}^{*} \geq c_{k} \Delta(Q)$, where $c_{k}=4 / 27+2 c_{k-1} / 27$ and $c_{0}=4 / 27$. It is easy to verify that this sequence of results converges to $\mu_{Q}^{*} \geq 4 \Delta(Q) / 25$.

Corollary 2.2. Let $P$ be a non-convex simple polygon, such that the ratio between the area of a minimum-area enclosing ellipse of $P$ and the area of a maximum-area enclosed ellipse is at most $\beta$, for some constant $\beta \geq 1$. Then $\mu_{P}^{*} \geq 4 \Delta(P) /\left(25 \beta^{2}\right)$.
Proof: As in [3], except that we apply the improved bound of Theorem 2.1.

\section{$31 / 3 \leq c_{2}^{*} \leq 2 /(3 \sqrt{3})$}

As mentioned in the introduction, the average distance between the Fermat-Weber center of a disk $D$ (i.e., $D$ 's center) and the points in $D$ is $\Delta(D) / 3$, where $\Delta(D)$ is the diameter of $D$. This immediately implies that $c_{2}^{*} \geq 1 / 3$. We prove below that $c_{2}^{*} \leq 2 /(3 \sqrt{3})$.

We first state a simple lemma and a theorem of Jung that are needed for our proof.

Lemma 3.1. Let $R, Q$ be two (not-necessarily convex) disjoint objects, and let $p$ be a point in the plane. Then, $\mu_{(R \cup Q)}(p) \leq \max \left\{\mu_{R}(p), \mu_{Q}(p)\right\}$.

Proof:

$$
\begin{aligned}
\mu_{(R \cup Q)}(p) & =\frac{\int_{x \in R \cup Q}\|p x\| d x}{\operatorname{area}(R \cup Q)}= \\
& =\frac{\int_{x \in R}\|p x\| d x+\int_{x \in Q}\|p x\| d x}{\operatorname{area}(R)+\operatorname{area}(Q)}= \\
& =\frac{\operatorname{area}(R) \cdot \mu_{R}(p)+\operatorname{area}(Q) \cdot \mu_{Q}(p)}{\operatorname{area}(R)+\operatorname{area}(Q)} \leq \\
& \leq \frac{(\operatorname{area}(R)+\operatorname{area}(Q)) \max \left\{\mu_{R}(p), \mu_{Q}(p)\right\}}{\operatorname{area}(R)+\operatorname{area}(Q)} \leq \\
& \leq \max \left\{\mu_{R}(p), \mu_{Q}(p)\right\} .
\end{aligned}
$$

Theorem 3.2 (Jung's Theorem [5, 6]). Every set of diameter $d$ in $\mathbb{R}^{n}$ is contained in a closed ball of radius $r \leq d \sqrt{\frac{n}{2(n+1)}}$. In particular, if $R$ is a convex object in the plane, then the radius of the smallest enclosing circle $C$ of $R$ is at most $\Delta(R) / \sqrt{3}$, where $\Delta(R)$ is the diameter of $R$.

Theorem 3.3. For any convex object $R, \mu_{R}^{*} \leq$ $2 \Delta(R) /(3 \sqrt{3})$.

Proof: Let $R$ be a convex polygon. Let $C$ be the smallest enclosing circle of $R$, and let $o$ and $r$ denote $R$ 's center point and radius, respectively. Notice that $o \in R$, since $R$ is convex. We divide $R$ into 8 regions $R_{1}, \ldots, R_{8}$ by drawing four line segments through $o$, such that each of the 8 angles formed around $o$ is of $45^{\circ}$; see Figure 3(a). Clearly, for each $R_{i}, o \in R_{i}$ and $\Delta\left(R_{i}\right) \leq r$.

We first prove that for each region $R_{i}, \mu_{R_{i}}(o) \leq$ $2 \Delta\left(R_{i}\right) / 3$. (This is done by adapting the proof of Lemma 3.1 of Aronov et al. [1].) Consider Figure 3(b). Let $p \in R_{i}$ be the farthest point from $o$. Draw the circular sector ocd centered at $o$ of radius $\|o p\|$. Let $a$ and $b$ be as in Figure 3(b). Let $f$ be the point on the arc $c d$, such that the regions $Q_{1}$ and $Q_{2}$ obtained by drawing the segment of are of equal area. $\left(Q_{1}\right.$ is the region $o x b$ and $Q_{2}$ is the difference between the sector opf and the 


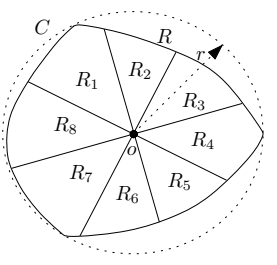

(a)

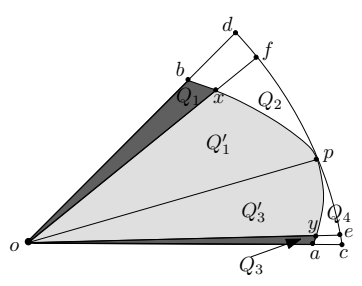

(b)
Figure 3: Illustrating the proof of Theorem 3.3.

region opx, where $x$ is the intersection point between of and the boundary piece $p b$.) Similarly, let $e$ be the point on the arc $c d$, such that the regions $Q_{3}$ and $Q_{4}$ obtained by drawing the segment oe are of equal area. $\left(Q_{3}\right.$ is the region oay and $Q_{4}$ is the difference between the sector oep and the region oyp, where $y$ is the intersection point between oe and the boundary piece $a p$.)

Now, on the one hand, since opb is convex, $x$ is the farthest point from $o$ in $Q_{1}$, and, on the other hand, $x$ is the closest point to $o$ in $Q_{2}$. Hence, any point in $Q_{2}$ is farther from $o$ than any point in $Q_{1}$. Thus we get that $\mu_{o p b}(o)=\mu_{\left(Q_{1}^{\prime} \cup Q_{1}\right)}(o) \leq \mu_{\left(Q_{1}^{\prime} \cup Q_{2}\right)}(o)=\mu_{o p f}(o)=$ $2\|o p\| / 3=2 \Delta R_{i} / 3$. We show that $\mu_{\text {oap }}(o) \leq 2 \Delta R_{i} / 3$ using the "symmetric" analysis. Since opb and oap are disjoint convex objects, then, by Lemma $3.1, \mu_{R_{i}}(o)=$ $\mu_{(\text {opb } \cup \text { oap })}(o) \leq 2 \Delta R_{i} / 3$.

We now show that $\mu_{R}(o) \leq 2 \Delta(R) /(3 \sqrt{3})$, immediately implying that $\mu_{R}^{*} \leq 2 \Delta(R) /(3 \sqrt{3})$. By Theorem 3.2, we know that $r \leq \Delta(R) / \sqrt{3}$. We also know that for each $R_{i}, \Delta\left(R_{i}\right) \leq r$. Thus, $\mu_{R_{i}}(o) \leq$ $2 \Delta\left(R_{i}\right) / 3 \leq 2 r / 3 \leq 2 \Delta(R) /(3 \sqrt{3})$.

We now apply Lemma 3.1 to obtain that

$$
\begin{gathered}
\mu_{R}(o) \leq \max \left\{\mu_{\left(R_{1} \cup R_{2} \cup R_{3} \cup R_{4}\right)}(o), \mu_{\left(R_{5} \cup R_{6} \cup R_{7} \cup R_{8}\right)}(o)\right\} \leq \\
\leq \max \left\{\max \left\{\mu_{\left(R_{1} \cup R_{2}\right)}(o), \mu_{\left(R_{3} \cup R_{4}\right)}(o)\right\}\right. \\
\left.\max \left\{\mu_{\left(R_{5} \cup R_{6}\right)}(o), \mu_{\left(R_{7} \cup R_{8}\right)}(o)\right\}\right\} \leq \\
\quad \vdots \quad \max \left\{\mu_{R_{1}}(o), \mu_{R_{2}}(o), \mu_{R_{3}}(o), \mu_{R_{4}}(o)\right. \\
\left.\mu_{R_{5}}(o), \mu_{R_{6}}(o), \mu_{R_{7}}(o), \mu_{R_{8}}(o)\right\} \leq \\
\leq 2 \Delta(R) /(3 \sqrt{3}) .
\end{gathered}
$$

Corollary 3.4. Let $P$ be a convex $n$-gon. Then one can compute in linear time a point $p$, such that $\mu_{P}(p) \leq$ $\frac{25}{6 \sqrt{3}} \mu_{P}^{*}$.

Proof: We apply Megiddo's linear-time algorithm for computing the smallest enclosing circle $C$ of $P$ [7]. Let $p$ denote the center of $C$, then, by Theorem 2.1

$$
\frac{\mu_{P}(p)}{\mu_{P}^{*}} \leq \frac{2 \Delta(P) /(3 \sqrt{3})}{4 \Delta(P) / 25}=\frac{25}{6 \sqrt{3}} .
$$

Corollary 3.4 gives us a very simple linear-time constant-factor approximation algorithm for finding an approximate Fermat-Weber center in a convex polygon. A less practical linear approximation scheme for finding such a point was presented by Carmi et al. [3].

\section{Application}

We consider the load balancing problem studied by Aronov et al. [1]. Let $D$ be a convex region and let $\mathcal{P}=\left\{p_{1}, \ldots, p_{m}\right\}$ be a set of $m$ points representing $m$ facilities. The goal is to divide $D$ into $m$ equal-area convex regions $R_{1}, \ldots, R_{m}$, so that region $R_{i}$ is associated with point $p_{i}$, and the total cost of the subdivision is minimized. The cost $\kappa\left(p_{i}\right)$ associated with facility $p_{i}$ is the average distance between $p_{i}$ and the points in $R_{i}$, and the total cost of the subdivision is $\sum_{i} \kappa\left(p_{i}\right)$.

Assuming $D$ is a rectangle that can be divided into $m$ squares of equal size, Aronov et al. present an $O\left(\mathrm{~m}^{3}\right)$ time algorithm for computing a subdivision of cost at most $(8+\sqrt{2 \pi})$ times the cost of an optimal subdivision. By applying Theorem 2.1 in the analysis of their algorithm, we obtain a better approximation ratio, namely, $\left(\frac{29}{4}+\sqrt{2 \pi}\right)$. For further details, see the full version of this paper.

\section{References}

[1] B. Aronov, P. Carmi, and M.J. Katz. Minimum-cost load-balancing partitions. Proc. 22nd ACM Symp. on Computational Geometry, pages 301-308, 2006.

[2] C. Bajaj. The algebraic degree of geometric optimization problems. Discrete and Comput. Geom., 3 (1988), 177-191.

[3] P. Carmi, S. Har-Peled, and M.J. Katz. On the Fermat-Weber center of a convex object. Comput. Geom. Theory and Appls, 32(3) (2005), 188-195.

[4] S.P. Fekete, J.S.B. Mitchell, and K. Weinbrecht. On the continuous Weber and $k$-median problems. Proc. 16th ACM Symp. on Computational Geometry, pages 70-79, 2000.

[5] H.W.E. Jung. Über die kleinste Kugel, die eine räumliche Figur einschließt. J. Angew. Math. 123 (1901), 241-257.

[6] H.W.E. Jung. Über den kleinsten Kreis, der eine ebene Figur einschließt. J. Angew. Math. 137 (1910), 310-313.

[7] N. Megiddo. Linear-time algorithms for linear programming in $\mathbb{R}^{3}$ and related problems. SIAM Journal on Computing, 12(4) (1983), 759-776.

[8] G. Wesolowsky. The Weber problem: History and perspectives. Location Science, 1(1) (1993), 5-23. 